\title{
The effects of lithographic residues and humidity on graphene field effect devices
}

\author{
BEHIYE BOYARBAY KANTAR ${ }^{1}$, MUHITTIN ÖZTÜRK ${ }^{2}$ and HIDAYET ÇETİN ${ }^{3, *}$ \\ ${ }^{1}$ Department of Physics, Science Faculty, Erciyes University, Kayseri 38039, Turkey \\ ${ }^{2}$ Department of Physics, Niğde University, Graduate School Natural and Applied Sciences, Niğde 51240, Turkey \\ ${ }^{3}$ Department of Physics, Art and Science Faculty, Bozok University, Yozgat 66900, Turkey
}

MS received 15 March 2016; accepted 2 July 2016

\begin{abstract}
Recently, unknown-manner changes in charge neutrality point (CNP) positioning were ascribed to humidity at graphene field effect transistors (GFETs). While the exact means of humidity interacting with hydrophobic graphene remains unknown, this work examines pristine and lithographic-process-applied graphene surfaces with surface enhanced Raman spectra (SERS). SERS analysis shows that the lithographic-process-applied graphene does not have the same properties as those of pristine graphene. Furthermore, this study has experimentally investigated the effect of humidity on the transfer characteristics of GFET and proposed a model to explain the formation of asymmetric $I_{\mathrm{DS}}-V_{\mathrm{bg}}$ branches in accordance with the SERS results and humidity responses.
\end{abstract}

Keywords. Graphene; SERS; GFET; humidity.

\section{Introduction}

Graphene is a two-dimensional material that was experimentally discovered for the first time by Novoselov et al [1]. It has unconventional properties such as finite conductivity when the charge carrier density is zero, an anomalous room temperature quantum Hall effect, linear electron dispersion at the vicinity of the Fermi level and electrically tuneable doping [2,3]. Its carrier type and the concentration modulation achieved by applying external gate voltage make it a promising material for future electronic devices [4]. There are a few problems to counter these unique benefits of manufacturing a device made from graphene. One of them is unknown charge neutrality point (CNP) positioning. Generally, the CNP is modified after the applied fabrication process. In graphene device fabrication, the control of the CNP assists in controlling the doping type and density. Several studies have been conducted to explain CNP shifting [5-8]. After fabrication of the graphene field effect transistor (GFET), non-zero CNP positioning is generally observed at the drain-to-source current vs. gate voltage $\left(I_{\mathrm{DS}}-V_{\mathrm{bg}}\right)$ characteristics, and the humidity is seen as a contributing factor of non-zero CNP positioning. Furthermore, $I_{\mathrm{DS}}-V_{\mathrm{bg}}$ characteristics could show an asymmetry with non-linear p- and n-branches when GFET has been exposed to a reactive environment. Thus, the first aim of this study is to investigate the effect of humidity on $\mathrm{CNP}$ and the formation of asymmetric branches.

According to a theoretical study, graphene is not sensitive to water adsorbed perturbation [9]. On the other hand, graphene is already highly hydrophobic, which prevents

*Author for correspondence (hidayet.cetin@bozok.edu.tr) water molecules from binding with graphene [10]. In this case, understanding how humidity can affect the electrical characteristics of GFETs is important. For this purpose, we have investigated the graphene's surface and the GFET's response to humidity. Dan et al [11] investigated the effect of lithographic contamination on transfer characteristics and the GFET sensing mechanism. They found a layer of $\sim 1 \mathrm{~nm}$ thickness by using atomic force microscopy (AFM) on the e-beam lithography and lift-off applied graphene surface. They only investigated the GFET surface by AFM, which means that spectral investigations are needed to verify the hypothesis of resist contamination. Thus, photolithography and lift-off applied graphene surface have been examined, and the presence of resist contamination is shown by SERS, which is a powerful method [12] to explore sample surface for the first time. The obtained results are not only important in terms of explaining water-graphene interaction, but also in understanding the mechanisms of detection of adsorbents by graphene sensors.

\section{Experimental}

In this study, one-atom thick graphene was prepared by mechanical exfoliation of natural graphite on a $\mathrm{SiO}_{2}$ $(285 \mathrm{~nm}) / \mathrm{Si}(450 \mu \mathrm{m})$ substrate. The exfoliation was continued until flakes were nearly invisible in the optical microscope. Thin graphene flakes are identified under an optical microscope with $1000 \times$ magnification. For SERS analysis, two graphene samples were prepared through a mechanical exfoliation of graphite. The photolithographic process was applied to one of the graphene samples with AZ5214 photoresist. Acetone was used at the lift-off process. A 785-nm 
excitation laser and 6-nm thermally evaporated Au nanoparticles were used for the SERS measurements. The heating effects can be neglected with $35 \mu \mathrm{W}$ incident power.

$\mathrm{Au} / \mathrm{Cr}(30 / 20 \mathrm{~nm})$ electrical contacts were fabricated at the designated locations using standard photolithographic techniques and thermal evaporation. The device channel length was determined to be $6 \mu \mathrm{m}$. As can be seen in figure 1a and $\mathrm{b}$, whole GFET response measurements were done at $25^{\circ} \mathrm{C}$ in a stainless-steel closed chamber, which was equipped with a calibrated humidity sensor. The instruments that were used to measure electrical response and electronic reading parts of the humidity sensor were out of the chamber to protect the effect of humidity. Thus, only GFET and calibrated humidity sensor were exposed to humidity in the closed chamber.
The humidity was supplied to the chamber by another system that generates cold water vapour from ultrapure water (18.2 M $\Omega$ ). The chamber's humidity level was controlled by adjusting both the amount of steam and the chamber pressure. The electrical characteristics of the graphene FET were measured with a Keithley 2612 using a feedthrough fixed on the chamber.

\section{Results and discussion}

SERS is one of the most important means of trace detection due to its dramatically increased Raman scattering signals with enhancement factors of $10^{4}-10^{8}$ over normal scattering.
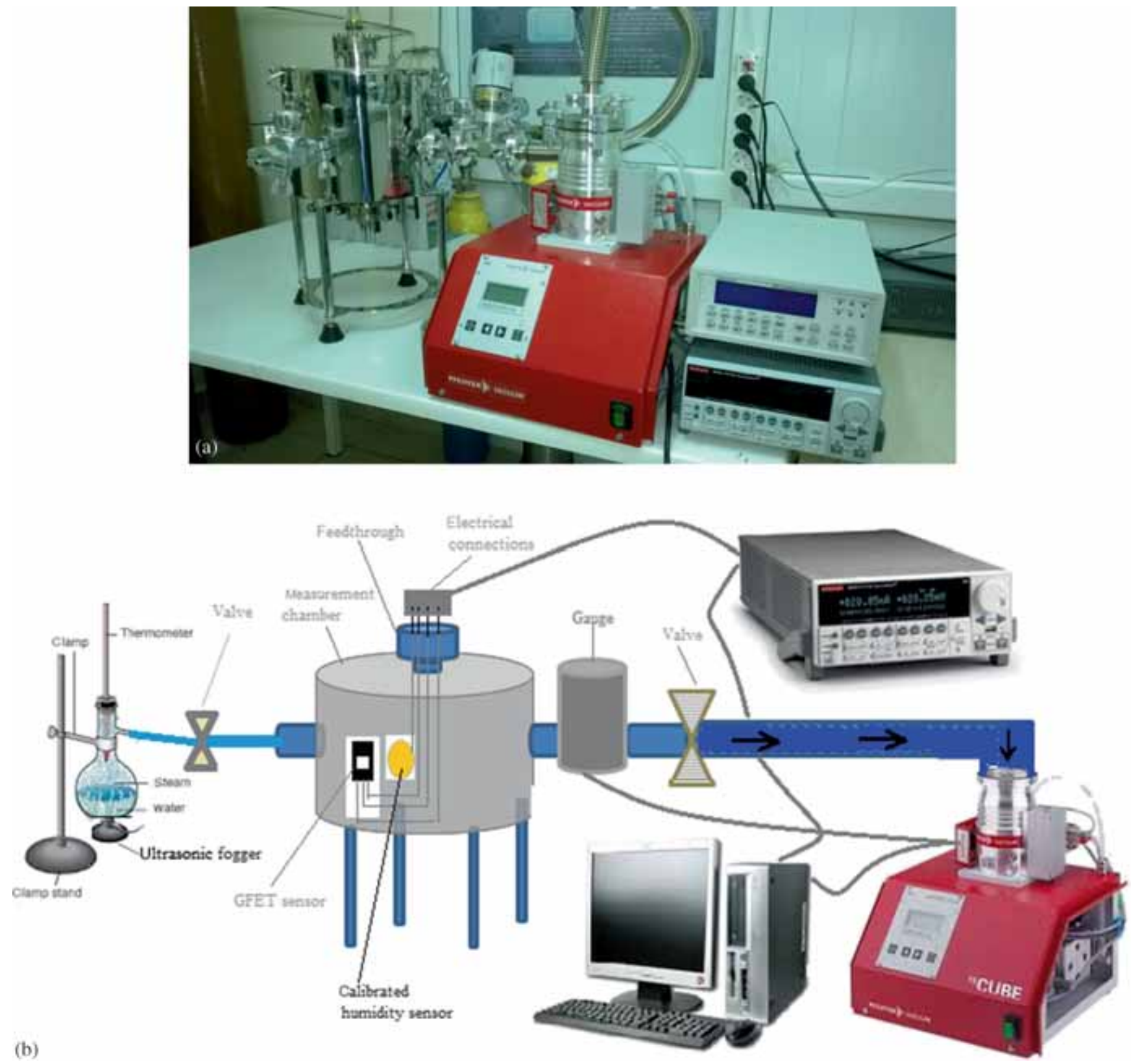

Figure 1. (a) The photograph and (b) schematic picture of the measurement system. 


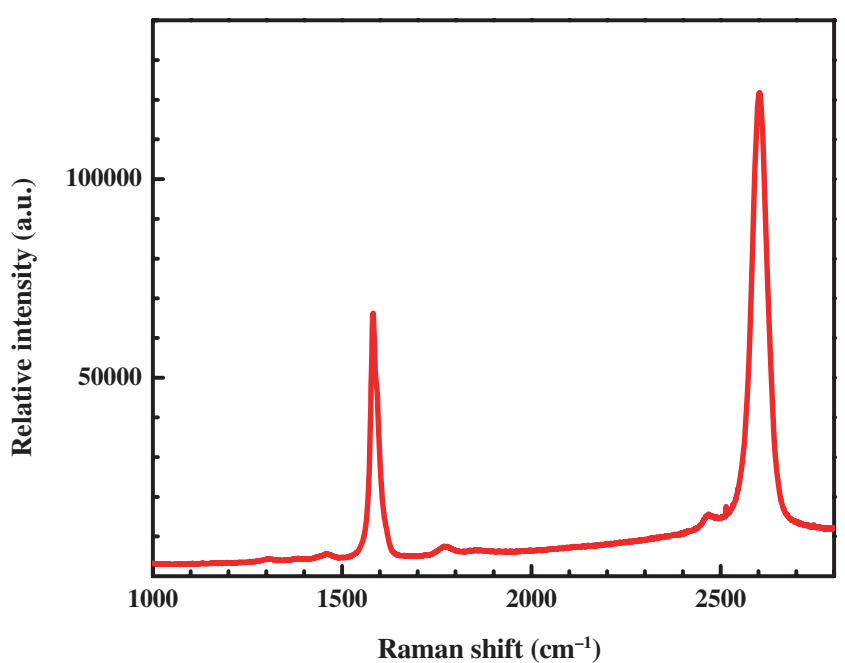

Figure 2. SERS of the pristine graphene.

For a single molecule, the enhancement factor could be even up to $10^{14}[13,14]$. Because of the enormous enhancement factor, SERS can be used as a powerful tool for exploring surface ingredients. This study used SERS analysis to show the difference between pristine and lithography-applied graphene. While Raman signal cannot be obtained because of intense fluorescence background at $785 \mathrm{~nm}$ excitation wavelength, SERS signal provides information about surfaces. Figure 2 shows the spectrum of pristine graphene. As it demonstrates, there are two clear, characteristic peaks in the figure. The $\mathrm{G}$ line is close to $1580 \mathrm{~cm}^{-1}$, and the $2 \mathrm{D}$ is around $2600 \mathrm{~cm}^{-1}$. The $\mathrm{D}$ line normally relates graphene defects, but no $\mathrm{D}$ band is observed around $1350 \mathrm{~cm}^{-1}$ in this sample. The intensity ratio value of $I_{2 \mathrm{D}} / I_{\mathrm{G}}$ is $\sim 2$ in a single layer of graphene [15].

To determine the SERS spectrum of photoresist residue, photolithography was applied onto a blank $\mathrm{SiO}_{2} / \mathrm{Si}$ sample. After the developing procedure, photoresist was removed from the surface. Figure 3a demonstrates SERS spectrum of the residue. A peak at $2120 \mathrm{~cm}^{-1}$ and peaks between 1100 and $1600 \mathrm{~cm}^{-1}$ can be seen in the SERS spectrum. After measuring of SERS spectrum of photoresist residue, we obtained SERS spectrum from photolithography and lift-off applied graphene which is on $\mathrm{SiO}_{2} / \mathrm{Si}$ substrate. The spectrum is shown in figure $3 \mathrm{~b}$. When figure $3 \mathrm{~b}$ is carefully examined, it is clearly seen that it includes both resist residue and characteristic peaks of graphene. The other noticeable point is that intensity of $G$ and $2 D$ peaks in figure 2 decreases with existing photoresist residue in figure $3 \mathrm{~b}$. Furthermore, the intensity ratio value of $2 \mathrm{D}$ to $\mathrm{G}$, which is a determinative factor of number of the graphene layer, was found to be $<1$. The new peaks at around 1290 and $2120 \mathrm{~cm}^{-1}$ and new shoulders near the $\mathrm{G}$ line emphasize a modified graphene surface. After the lithographic process, the graphene does not have pristine properties according to the SERS results. The new peaks and shoulders indicate the existence of the residue.
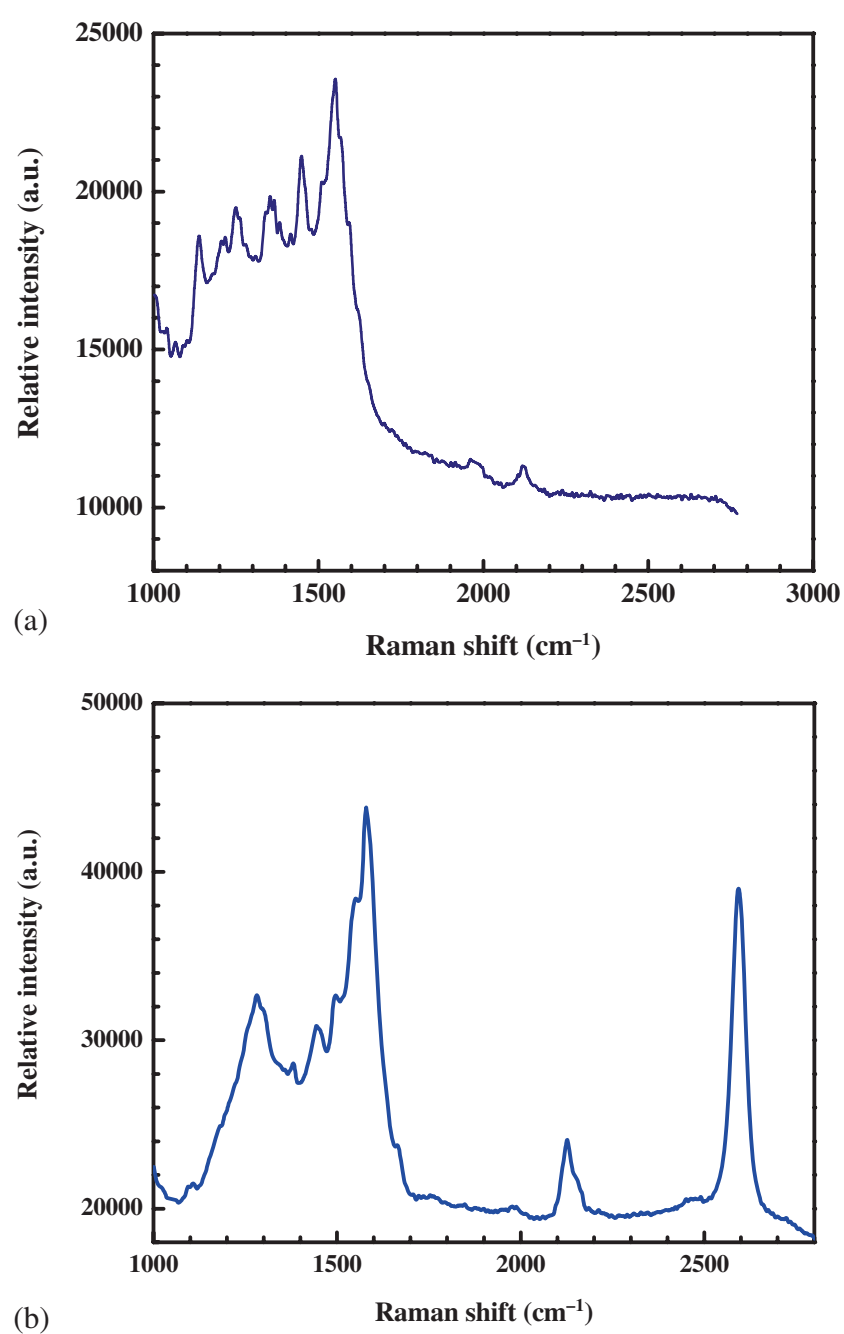

Figure 3. (a) SERS of photolithography and lift-off applied $\mathrm{SiO}_{2}$ surface. SERS results show that after the photolithography, resist residue remains on the substrate surface. (b) SERS of photolithography and lift-off applied graphene. After the photolithography, new peaks arise at $1279,1446,1494,1548$ and $2124 \mathrm{~cm}^{-1}$.

The photoresist residue was examined with a scanning electron microscope (SEM), an optical microscope as well as the SERS method. In figure 4a, SEM image of the pristine graphene layer is shown. The image shows single-layer graphene as well as the layered part. An ETD detector was used for the image. When the image is carefully examined, any residue cannot be seen for the pristine graphene. Figure $4 \mathrm{~b}$ shows SEM image of the GFET. In figure 4c, graphene channel of the GFET is given with further magnification that is the same as that of the image of the pristine graphene. The left side in the figure is graphene and the other is $\mathrm{SiO}_{2}$ substrate. Comparing figure $4 \mathrm{a}$ and $\mathrm{c}$ to each other, spotted residue can be slightly seen on the graphene surface in figure 4c. However, the residue layer cannot be observed in the optic image presented in figure 5a.

Besides all these, it is possible to make the covered residue visible. This is another evidence for the presence of residue. When the sample was exposed to UV light $(360 \mathrm{~nm})$ for 

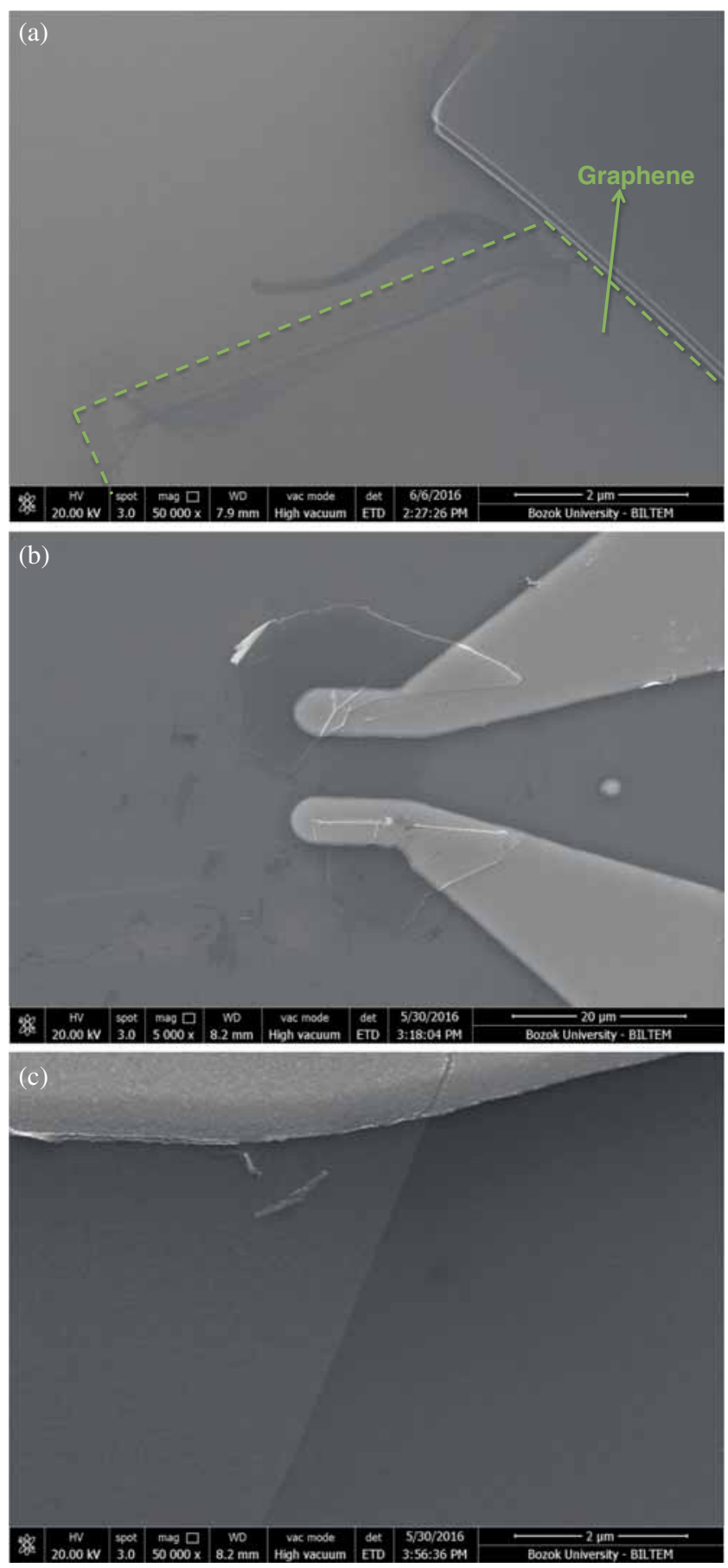

Figure 4. (a) SEM image of pristine graphene layer. Graphene layer with wrinkled edge could be easily noticed. (b) SEM image of the GFET. (c) SEM image of GFETs' graphene channel. Dotshaped lithographic residue could be seen on the graphene channel.

$60 \mathrm{~min}$, inhomogeneous surface residue appeared. The inhomogeneous surface residue is shown in figure $5 b$ at $100 \times$ optical magnification. It is thought that surface residue is a monomer that cannot be a polymer during the photolithography process or monomer that cannot be removed during the lift-off process. The applied UV light for $60 \mathrm{~min}$ causes polymerization with large chains. After the UV exposure

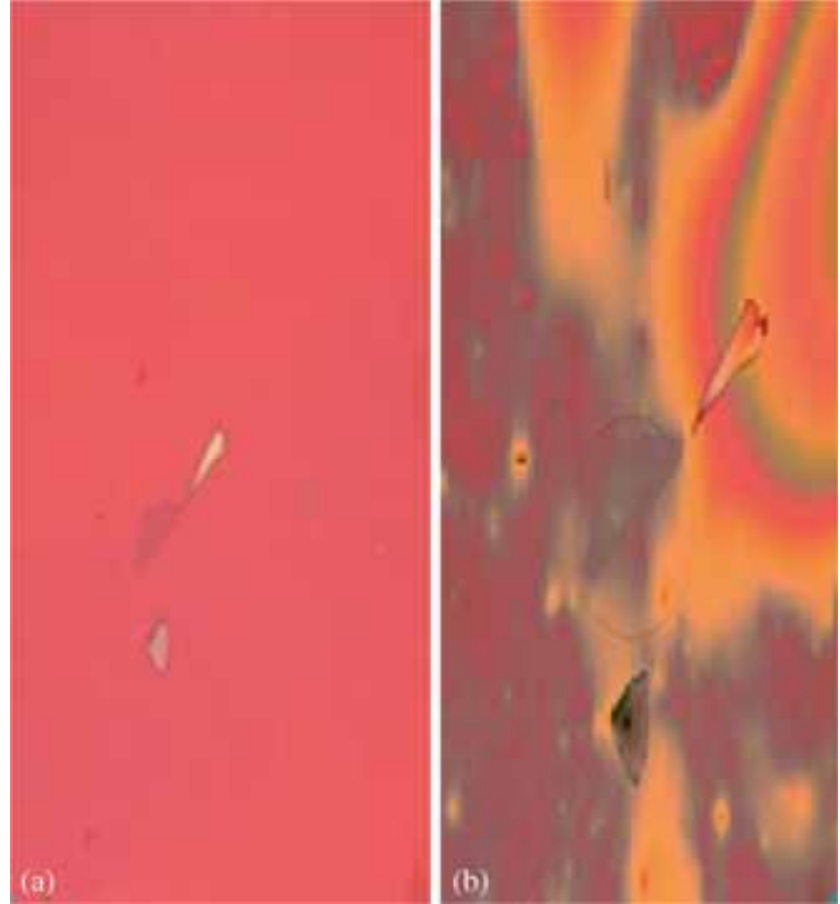

Figure 5. (a) Optical microscope $(20 \times)$ image of photolithography and lift-off applied graphene. The photoresist residue cannot be seen in the image. (b) To make visible the residue, UV and $\mathrm{O}_{2}$ gas were applied to the sample. After the applied procedure, residue can be clearly seen using optical microscope.

procedure, the photoresist residue is visible. The photoresist residue may be one of the reasons of charge doping that shifts the CNP to a non-zero position. The pristine graphene is highly hydrophobic, but the lithography-applied graphene has a modified surface that can interact with humidity. Thus, humidity-sensitive characteristics can be expected from GFETs.

In figure 6, the transfer characteristics of the GFET are shown under atmosphere and vacuum conditions for $V_{\mathrm{DS}}=$ $10 \mathrm{mV}$. After deposition of the metal contacts, the first measurement (indicated by the black line in figure 5) is performed in atmosphere of $34 \%$ humidity. According to the graph, the graphene is p-type doped in the atmospheric condition because the CNP appears at $27 \mathrm{~V}$. Atmospheric gases, $\mathrm{Cr} / \mathrm{Au}$ metal contacts $[16,17]$ and humidity [5-18] cause p-type doping. Next the graphene sample is isolated from the atmospheric environment within a closed vacuum chamber. The chamber is set to a $6.8 \times 10^{-3}$ mbar vacuum, and the humidity is measured as $<1 \%$ by the humidity sensor. The $I_{\mathrm{DS}}-V_{\mathrm{bg}}$ measurement was repeated. The position of CNP in this environment was observed at $+9 \mathrm{~V}$ with $18 \mathrm{~V}$ shifting. The graphene device remains as hole doped when the humidity value is reduced from 34 to $1 \%$. The decreasing pressure results in decreasing humidity and atmospheric gases. When the vacuum is improved to a $3 \times 10^{-3}$ mbar value, a new measurement result appeared as indicated by the green line in figure 6. The new CNP location is about $-6 \mathrm{~V}$ in the $V_{\mathrm{bg}}$ axis, and n-type doping appears. Graphene includes both hole 
and electron doping under atmospheric condition. After removing the hole doping, the resulting electron-doped graphene is associated with majority and minority carriers at conventional semiconductors. However, n-type doping factors cannot be removed by vacuum, which indicates that non-volatile factors cause n-type doping. The lithographic residue, which is detected with separate techniques as presented in figures $3 \mathrm{~b}, 4 \mathrm{c}$ and $5 \mathrm{~b}$, will undoubtedly be an effective factor for doping.

Figure 7 shows the $I_{\mathrm{DS}}-V_{\mathrm{bg}}$ characteristics of the GFET under various humidity values for $V_{\mathrm{DS}}=1 \mathrm{mV}$. For this experiment, firstly the chamber was pumped to the value of $<3 \times 10^{-3}$ mbar and then fixed at $3.6 \times 10^{-3}$ mbar pressure by supplying ultra-pure water vapour. The first result of the measurements, which is shown as a blue line, was obtained. At this pressure, the position of CNP is near the $0 \mathrm{~V}$ gate voltage

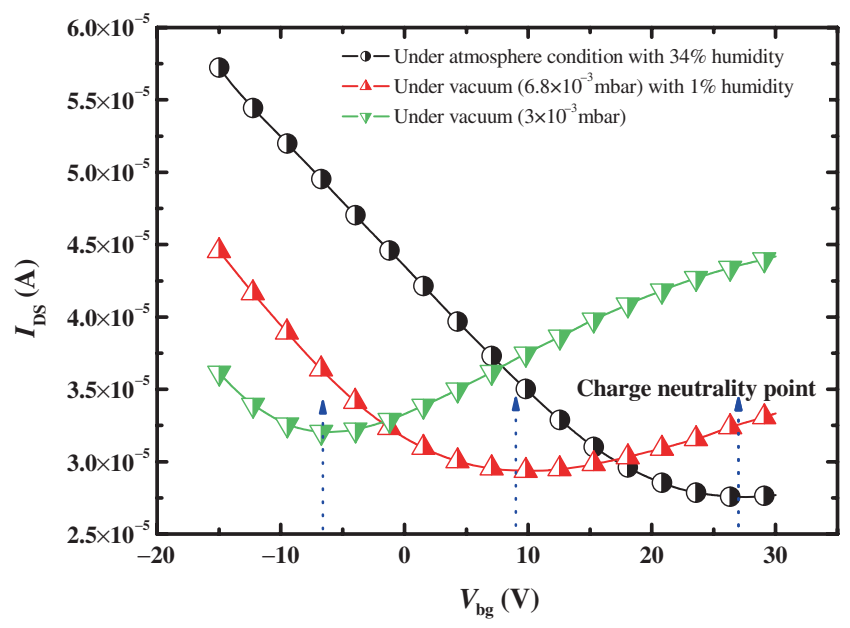

Figure 6. $I_{\mathrm{DS}}-V_{\mathrm{bg}}$ characteristics of the GFET at different atmosphere pressure and humidity conditions. Noticeable CNP shifting appears when different pressure are applied to the measurement chamber.

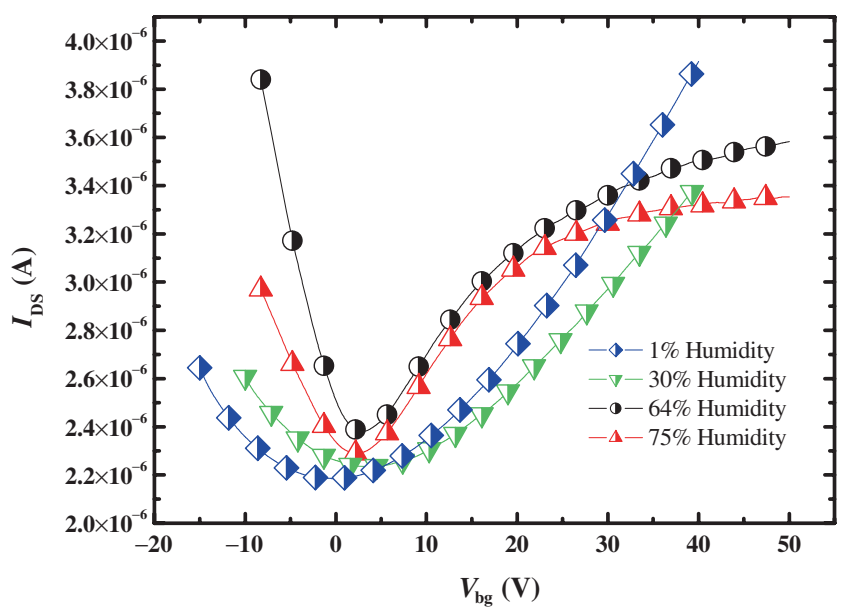

Figure 7. The electrical characteristics of the GFET under various humidity values. Increasing humidity changes CNP and/or the slope of the curves. As can be seen, 64 and $75 \%$ humidity values significantly change curve shapes. value. After the first measurement, the controlled manner ultra-pure water vapour is supplied to the chamber. When the humidity value reached $30 \%$, the $I_{\mathrm{DS}}-V_{\mathrm{bg}}$ measurement was repeated. The result obtained is indicated by the green line. It is clearly seen that supplying water vapour causes p-type doping and shifts CNP to $+5 \mathrm{~V}$. In addition to the shifting of CNP, the slope of the $I_{\mathrm{DS}}-V_{\mathrm{bg}}$ curve also changes. When the chamber humidity is increased to $64 \%$, a considerable change is seen in the curve. An asymmetry between p- and n-branches arises. However, the increasing humidity shifts the CNP to negative gate voltage values rather than to positive. This case is contrary to the expectation that p-type doping rises with increasing humidity. When humidity is increased to $75 \%$, the asymmetric curve is similar to that of $64 \%$ humidity, and the CNP remains unchanged. Whole experimental data in figures 6 and 7 show that humidity does not always cause the CNP shifting. The low-level humidity causes the CNP shift to the expected direction and the further humidity causes changing the shape of the $I_{\mathrm{DS}}-V_{\mathrm{bg}}$ curve. With the 64\% humidity, the CNP shift is unexpected. When figure $5 \mathrm{~b}$ is viewed, intensely residue covered regions and clean regions are clearly visible. These regions indicate inhomogeneous residue distribution. A water molecule can easily hold on the area where there is resist residue. Thus, spatially inhomogeneous molecule holding takes place on the graphene surface. Charge transfer could be effective for graphene's doping. The spatially inhomogeneous charge transfer causes high- and low-doped regions on graphene.

After observing the transfer characteristics at different humidities, the changing of transfer characteristics with humidity may bring to mind that the contact metal could be affected by humidity. To investigate contact metal quality at different humidities, we have used a Transfer Length Method technique. In the experiment, we made $\mathrm{Cr} / \mathrm{Au}(20 / 30 \mathrm{~nm})$ contacts on $\mathrm{Si} / \mathrm{SiO}_{2}$ substrate. In the experiment, the metal thin film was exposed to humidity for at least $10 \mathrm{~min}$ in a stainless-steel chamber. The chamber humidity value was fixed to values of $6,26,46,64$ and $81 \%$. In figure 8 ,

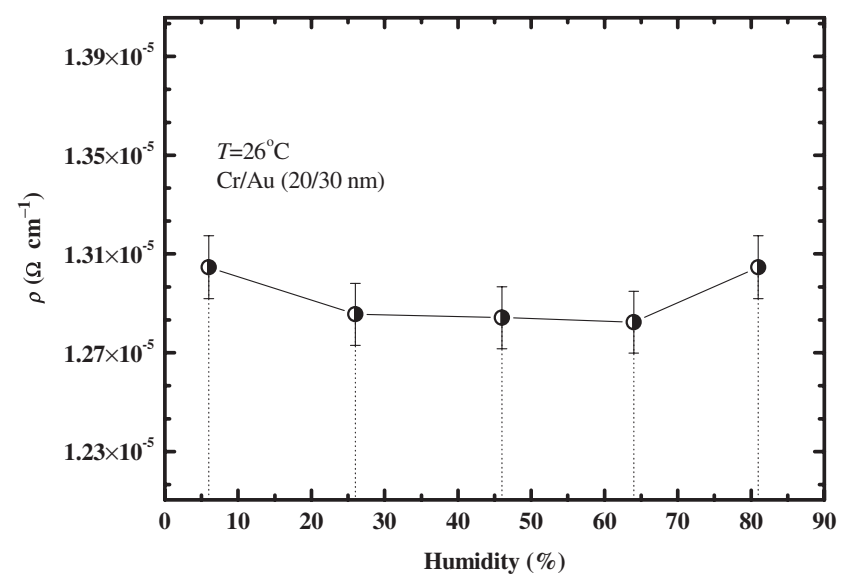

Figure 8. Resistivity of the $\mathrm{Cr} / \mathrm{Au}(20 / 30 \mathrm{~nm})$ contacts at different humidity values. $\mathrm{Cr} / \mathrm{Au}$ contacts show stable characteristic against to humidity in the frame of experimental error. 
$\mathrm{Cr} / \mathrm{Au}(20 / 30)$ thin film resistivity is shown at different humidity values. According to the results, $\mathrm{Cr} / \mathrm{Au}$ contact has very stable resistivity at different humidity values in the frame of experimental errors. Thus, the changing of the characteristics is attributed to charge transfer to graphene channel. In addition to charge transfer, a relatively high dipole moment of water molecule is the other noticeable factor. A water molecule has a polar structure and can be thought of as a dipole, which generates an electric field. In figure 9a, the electric field generated by water molecule and the electric field generated by gate voltage are shown schematically. As can be seen in figure $9 b$, the field generated by water molecule penetrates into the graphene at the channel region of GFET, which leads to doping. The gate-voltage-generated field and the additional dipole field determine the location of the CNP. If spherical coordinates are chosen so that $p$ lies at the origin, the electric field of a dipole is given by

$$
\boldsymbol{E}(r, \theta)=\frac{p}{\left(4 \pi \epsilon_{0} r^{3}\right)}(2 \cos \theta \hat{r}+\sin \theta \hat{\theta}),
$$

where $p$ is the dipole moment, $r$ the distance from the dipole, $\theta$ the polar angle and $\epsilon_{0}$ the permittivity of free space [19]. If $\mathrm{G}$ and $2 \mathrm{D}$ peaks of graphene can be observed by SERS measurements at photoresist-residue-coated graphene, the photoresist residue thickness must be very small. Revealing the effect of the electric field on the water molecule is

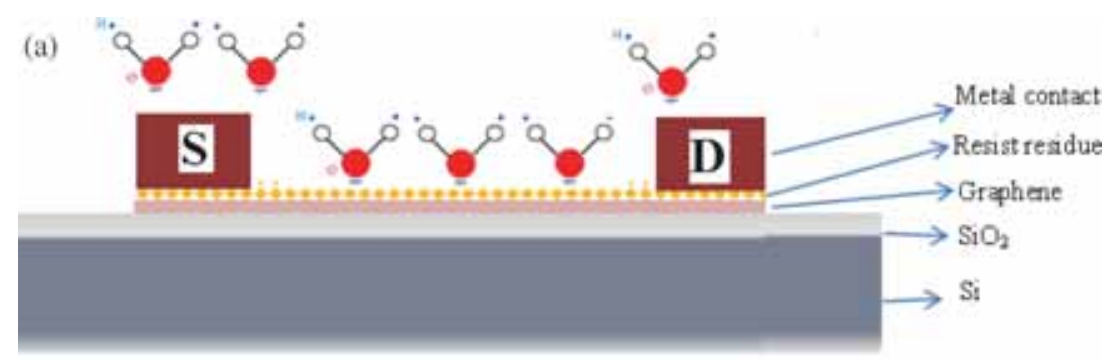

(b)

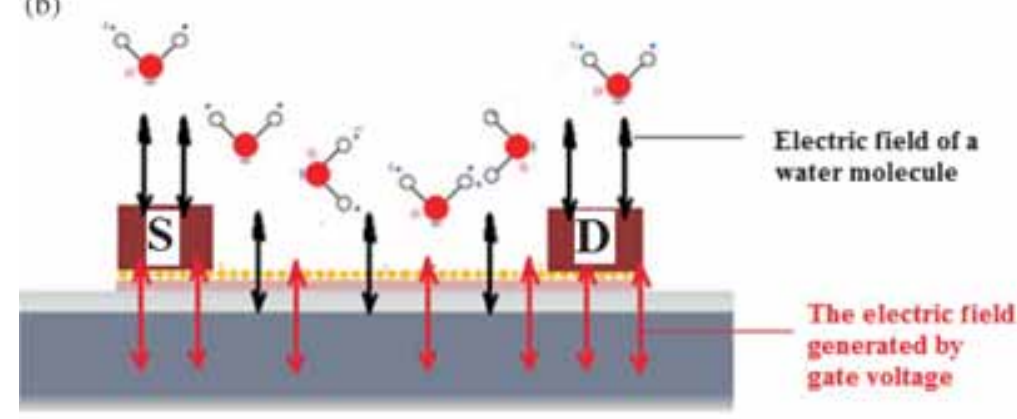

(c)

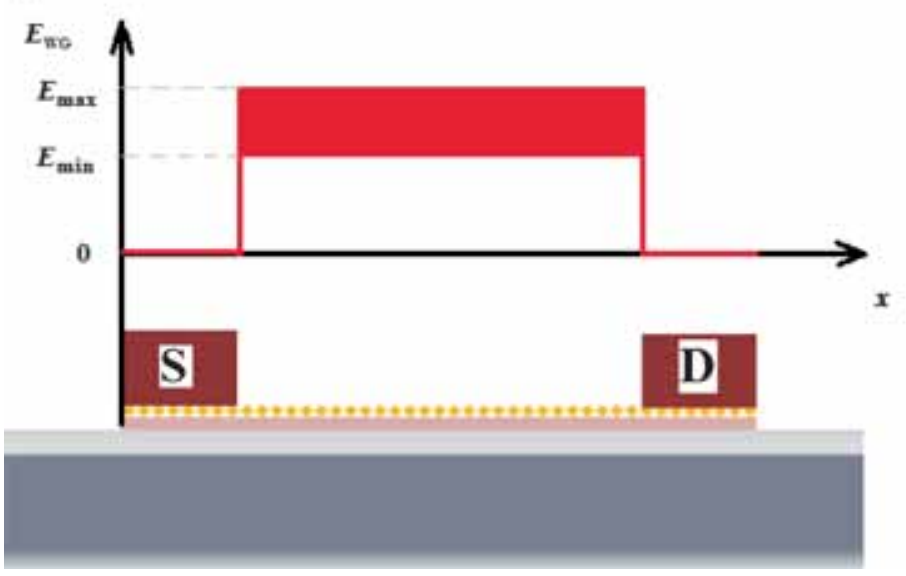

Figure 9. (a) Schematic view of the GFET structure. SERS results show that resist residue covers the graphene surface. (b) While the electric field of water molecules can reach the graphene channel, it does not affect graphene at the bottom of the metal contacts. The electric field is screened by metal contacts. (c) At the channel, electric field generated by water molecules is between $E_{\max }$ and $E_{\min }$ values depending on dipoles angle. The field of water molecules is zero under metal contacts $(20 / 30 \mathrm{~nm} \mathrm{Cr} / \mathrm{Au})$ due to screening the field by metal contacts. 
contingent on a few givens. Assuming a monolayer-watermolecule coating and using a water dipole moment value of $p=6.2 \times 10^{-30} \mathrm{C} \mathrm{m}^{-1}$, the resist dielectric constant $\epsilon=4$ and $r=1 \mathrm{~nm}$ in equation (1), and the angle-dependent electric field values $E_{\max }=1.1 \times 10^{8} \mathrm{~V} \mathrm{~m}^{-1}$ and $E_{\min }=$ $5.5 \times 10^{7} \mathrm{~V} \mathrm{~m}^{-1}$ can be obtained. These values easily rise up to $10^{10} \mathrm{~V} \mathrm{~m}^{-1}$ with thinner residue. Moser et al [20] investigated graphene surface with electrostatic force microscopy and found that water molecules form a dipole layer, which generates an electrical field as large as $10^{9} \mathrm{~V} \mathrm{~m}^{-1}$ on a graphene surface. This value supports our proposal. On the other hand, the effect of the dipole field cannot homogenously reach the entire graphene surface because of two reasons. The first reason is resist residue and its thickness inhomogeneity. When a water molecule hangs on the residue, a residue-thickness-dependent electric field affects the electrical doping of graphene. Thinner residue on graphene resulted in bigger electric fields. The residue' inhomogeneity gives rise to relatively high and low charge density regions on the graphene. The second reason is the screening of the electric field of the dipole by contact metals. The electric field screening may be one of the most important factors causing inhomogeneous charge distribution on the graphene. In figure $9 \mathrm{c}$, the field generated by the dipole indicated by black vertical lines and gate-voltage-generated field indicated by red lines are shown. As can be seen in figure 9c, the contactmetal-coated graphene regions are not affected by the field. It is well known that graphene carrier concentration is tunable by an external field. The sum of the gate-voltage-generated field and the dipole field assigns the density of carrier concentration and its type. The regions with different carrier concentration like the $\mathrm{p}-\mathrm{n}$ junction give rise to asymmetric $I_{\mathrm{DS}}-V_{\mathrm{bg}}$ curves.

\section{Conclusion}

In conclusion, an explanation about the interaction between hydrophobic graphene and humidity has been proposed. For this purpose, pristine and lithography-applied graphene surfaces were investigated using SERS. After the lift-off process, there is resist residue that is optically invisible on the graphene channel according to our SERS results. Although the theoretical works show that graphene is hydrophobic, GFET with lithographic contaminations is sensitive to humidity. Water molecules interact with the residue, and molecules can bind to them. The electric dipoles of hanged molecules can dope the channel region of graphene under a resist contamination layer but do not bind to the graphene region under the metal contacts because of the effects of screening. The field-induced doping difference increases between channel and contact regions and asymmetric branches appear at $I_{\mathrm{DS}}-V_{\mathrm{bg}}$ characteristics.

\section{Acknowledgements}

We will never forget Aslı ŞİMŞEK, who was a master student at our group, and we would like to thank her for valuable contributions. We would like to thank ARGESAN for contribution in fabricating a mask aligner. This work was supported by the Scientific and Technical Research Council of Turkey (TÜBİTAK) under Grant no. 108T930.

\section{References}

[1] Novoselov K S, Geim A K, Morozov S V, Jiang D, Zhang Y, Dubonos S V, Grigorieva I V and Firsov A A 2004 Science 306666

[2] Novoselov K S, Geim A K, Morozov S, Jiang Katsnelson V D M I, Grigorieva I V, Dubonos S V and Firsov A A 2005 Nature 438197

[3] Novoselov K S, Jiang Z, Zhang Y, Morozov S V, Stormer H L, Zeitler U, Maan J C, Boebinger G S, Kim P and Geim A K 2007 Science 3151379

[4] Chen J H, Jang C, Xiao S, Ishigami M and Fuhrer M S 2008 Nat. Nanotechnol. 3206

[5] Schedin F, Geim A K, Morozov S V, Hill E W, Blake P and Katsnelson M I 2007 Nat. Mater. 6652

[6] Wei P, Liu N, Lee H R, Adijanto E, Ci L, Naab B D, Zhong J Q, Park J, Chen W, Cui Y and Bao Z 2013 Nano Lett. 131890

[7] Bartolomeo A D, Giubileo F, Santandrea S, Romeo F, Citro R, Schroeder T and Lupina G 2011 Nanotechnology 22275702

[8] Romero H E, Joshi P, Gupta A K, Gutierrez H R, Cole M W, Tadigadapa S A and Eklund P C 2009 Nanotechnology 20245501

[9] Wehling T O, Katsnelson M I and Lichtenstein A I 2008 Appl. Phys. Lett. 93202110

[10] Leenaerts O, Partoens B and Peetersz F M 2008 Phys. Rev. B 77125416

[11] Dan Y, Lu Y, Kybert N J, Luo Z and Johnson A T C 2009 Nano Lett. 91472

[12] Schedin F, Lidorikis E, Lombardo A, Kravets V G, Geim A K, Grigorenko A N, Novoselov K S and Ferrari A C 2010 ACS Nano 45617

[13] Nie S and Emery S R 1997 Science 2751102

[14] Michaels A M, Nirmal M and Brus L E 1999 J. Am. Chem. Soc. 1219932

[15] Lee J, Shim S, Kim B and Shin H S 2011 Chem. Eur. J. 172381

[16] Giovannetti G, Khomyakov P A, Brocks G, Karpan V M, van den Brink J and Kelly P J 2008 Phys. Rev. Lett. 101026803

[17] Lv R and Terrones M 2012 Mater. Lett. 78209

[18] Joshi P, Romero H E, Neal A T, Toutam V K and Tadigadapa S A 2010 J. Phys.: Condens. Matter 22334214

[19] Griffiths D J 1999 Introduction to electrodynamics (3rd edn) (New Jersey: Prentice Hall) ISBN 0-13-805326-X

[20] Moser J, Verdaguer A, Jimenez D, Barreiro A and Bachtold A 2008 Appl. Phys. Lett. 92123507 